\title{
Conciliación entre la vida familiar y laboral: Evaluación del programa IGUALA en una empresa minera en la región de Tarapacá
}

\author{
Sandra Leiva Gómez \\ Universidad Arturo Prat, Iquique, Chile. \\ Email: sandleiva@gmail.com
}

\author{
Andrea Comelin Fornes \\ Universidad Arturo Prat, Iquique, Chile. \\ Email: ancomelin@gmail.com
}

\begin{abstract}
Resumen: ${ }^{1}$ La presente investigación analiza la implementación del programa IGUALA en relación a las medidas de conciliación entre vida familiar y laboral en una empresa minera en la región de Tarapacá, Chile. Se discuten dos enfoques teóricos que sustentan tales políticas: el genderfare y el enfoque de derechos. Al evaluar las acciones implementadas por la empresa, se establece en primer lugar que éstas no son realizadas considerando las perspectivas teóricas necesarias para conciliar familia y trabajo, segundo que la compañía enfrentó varios obstáculos para implementar las acciones, lo que redundó en un escaso impacto para los beneficiarios, y tercero que, a pesar de ello, éstos se muestran conformes con las políticas de la empresa en esta materia. Estos hallazgos traslucen las debilidades de la compañía al implementar las medidas de conciliación, pues se acercan más a un diagnóstico que a una efectiva política pública que tienda a mejorar la conciliación familia-trabajo.

Palabras clave: conciliación vida familiar y laboral, política pública, género, programa IGUALA, Chile.
\end{abstract}

\section{Reconciling work and family life: Evaluation of the IGUA- LA program in a mining company in the Tarapacá Region}

\begin{abstract}
The focus of this study is the implementation of the IGUALA program in relation to measures for reconciling work and family life in a mining company in the Tarapacá Region of Chile. Two theoretical approaches that sustain such policies are discussed: genderfare and the rights approach. The evaluation of the actions implemented by the company determined that theoretical perspectives on reconciling family life and work were not considered; that the company faced several obstacles on the implementation of the actions which limited their impact for beneficiaries; and that the beneficiaries were satisfied with company policy in this area in spite of that limited impact. These findings highlight the company's weaknesses in terms of the introduction of reconciliation measures, as they are closer to an assessment than an effective public policy for improving family-labor reconciliation.
\end{abstract}


Keywords: Reconciling family and work life, social policy, gender, IGUALA program, Chile.

\section{Conciliação entre a vida familiar e laboral: Avaliação do programa IGUALA numa empresa mineira na região de Tarapacá}

Resumo: A presente pesquisa analisa a implementação do programa IGUALA com relação às medidas de conciliação entre vida familiar e laboral numa empresa minera na região de Tarapacá, Chile. Discutem-se dois enfoques teóricos que sustentam tais políticas: o genderfare e o enfoque de direitos. Ao avaliar as ações implementadas pela empresa, se estabelece em primeiro lugar que elas não são realizadas considerando as perspectivas teóricas necessárias para conciliar família y trabalho; segundo que a companhia enfrentou vários obstáculos para praticar as ações, o qual redundou num escasso impacto para os beneficiários, e terceiro que, apesar de isso, estas se mostram conformes com as políticas da empresa nesta matéria. Tais descobertas transluzem as debilidades da companhia ao aplicar as medidas de conciliação, pois se aproximam mais a um diagnóstico que a uma efetiva política pública que tenda a melhorar a conciliação família-trabalho.

Palavras-chave: conciliação, vida familiar e laboral, política pública, género, programa IGUALA, Chile.

$$
* * *
$$

La masiva incorporación de la mujer al mundo del trabajo torna indispensable que las tareas de cuidado y domésticas al interior del hogar sean realizadas no sólo con participación de hombres y mujeres ${ }^{2}$, sino también con una ineludible participación del Estado. La labor de cuidado no solamente debe ser resuelta en forma privada por las trabajadoras, pues constituye un problema de equidad cuya resolución recae en la sociedad en su conjunto (Montaño, 2010; Arriagada, 2007a, 2007b; Aguirre, 2007). Al respecto, la OIT (2009) propone la corresponsabilidad en las tareas de cuidado entre hombres y mujeres por una parte, y entre la familia, el Estado, el mercado y la sociedad por otra, evidenciándose la necesidad de implementar políticas de conciliación con corresponsabilidad. Sin embargo, las políticas públicas para la conciliación familia-trabajo se tornan insuficientes si se entiende que tal conciliación debe resolverse centrada en facilitar las tareas a "las mujeres", desplazando la corresponsabilidad. Una mayor satisfacción derivada de una política eficiente en el área de cuidado genera a su vez mayor compromiso laboral y menor ausentismo, lo que implica en último término un aumento en la productividad de la empresa (Abarca y Errázuriz; 2007; SERNAM, 2003). En nuestro país algunas empresas han implementado medidas de conciliación familia-trabajo impulsadas desde el sector público por el SERNAM a través del programa IGUALA, cuyo objetivo central es promover buenas prácticas laborales con equidad de género.

Una evaluación de las medidas de conciliación de este programa resulta útil para conocer en qué medida se ha alcanzado el ejercicio de los 
derechos de cuidado de hombres y mujeres en pro de una equidad de género. Si bien es cierto existe ya una evaluación del programa realizada por el propio gobierno (Orellana, 2010), no hay un estudio que evalúe específicamente la implementación de las medidas tendientes a conciliar la vida familiar y laboral de este programa. Tampoco existe un estudio que indague la apreciación que los propios beneficiaros tienen del programa. Precisamente tal es el aporte que esta investigación viene a realizar. Por lo tanto, el presente estudio tiene por objetivo indagar cómo han sido implementadas las medidas tendientes a lograr la conciliación entre la vida familiar y laboral en una empresa minera en la región de Tarapacá. Para ello se utiliza una metodología cualitativa consistente en la aplicación de entrevistas en profundidad a tres actores involucrados en el programa. De esta manera, se entrevistó a dos informantes clave del SERNAM, a un informante clave de la empresa privada que implementó el programa y a cuatro trabajadores de planta de la empresa en tanto beneficiarias/os de éste. La elección de los beneficiarios se realizó siguiendo el criterio de que tuviesen hijos menores de seis años, a fin de observar facilidades otorgadas por la empresa para conciliar la vida familiar y laboral. ${ }^{3}$ El análisis se realizó en una compañía minera, porque fue una de las empresas premiadas por el SERNAM por la implementación de este programa. A fin de resguardar la confidencialidad, tanto el nombre de la compañía minera como de los entrevistados han sido anonimizados. Para el análisis de las entrevistas se utilizó el Software MAXQDA, el que permite la codificación de la información y la construcción de categorías a partir de los relatos recogidos.

En la siguiente sección se entregarán algunas ideas clave a la hora de realizar políticas públicas para conciliar la vida familiar y laboral. Para analizar esta materia se examinarán posteriormente dos enfoques teóricos: el genderfare y el enfoque de derechos. El genderfare enfatiza que un Estado de Bienestar (welfare) que sea realmente equitativo requiere incorporar al análisis la variable de género (gender); en tanto el enfoque de derechos centra su atención en el derecho que toda persona tiene a ser cuidada. Enseguida se revisará brevemente el programa IGUALA, identificando sus características y objetivos a cumplir, entregando luego antecedentes de la evaluación que el Gobierno realizara de él. Posteriormente se darán a conocer los resultados principales de esta investigación, analizando en primer lugar las medidas para conciliar de acuerdo a los dos enfoques teóricos examinados, en segundo lugar indicando los principales obstáculos al implementar las acciones para conciliar y tercero, analizando las opiniones de beneficiarias y beneficiarios sobre tales medidas. Finalmente se entregarán las conclusiones del estudio.

\section{Políticas públicas para lograr la conciliación entre la vida familiar y laboral}

El debate público internacional ha puesto de relieve las políticas públicas conciliatorias. Así, la Conferencia de Beijing estableció que deben armonizarse las responsabilidades entre el trabajo y la familia por parte de 
hombres y mujeres. Por otra parte, la Convención sobre la Eliminación de Todas las Formas de Discriminación contra la Mujer señala que deben tomarse medidas para eliminar la discriminación contra la mujer y asegurar de esta forma una igualdad de derechos. Asimismo, el Convenio $\mathrm{N}^{\circ} 156$ de la Organización Internacional del Trabajo manifiesta la responsabilidad familiar como una tema que atañe no sólo a la familia (Lamus, 2008; CEPAL, 2012; Pautassi y Rico, 2011).

Las políticas de conciliación no solo permiten equilibrar la vida laboral y familiar. Por una parte son una vía para caminar hacia la equidad de género y por otra permiten transitar hacia la reparación de profundas desigualdades sociales, ya que sobre todo en América Latina las desigualdades de género se encuentran en el cimiento del círculo vicioso que reproduce la pobreza (Batthyany, 2009). Según Aguirre (2007) los cuidados deben constituirse en una política pública principalmente por tres razones: el cuidado se ha trasladado a las esferas privadas, ya sea al mercado o a la familia, mientras el Estado se desentiende de esta necesidad. En segundo lugar, existe un creciente aumento de los adultos mayores y por último, se observa un aumento de la actividad económica femenina. Por otra parte, una política pública de cuidado tiene que considerar una serie de funciones, ,entre las que se contemplan en primera instancia hacer las tareas de cuidado colectivas a través de la provisión de servicios, en vez de obligar a las mujeres a resolverlo mediante soluciones individuales que normalmente las perjudican. En segundo lugar es fundamental transferir recursos monetarios a las familias, reconociendo la función social del cuidado, el costo privado que implica y el valor que posee para la economía del país. Se debe además promover el cambio y la solidaridad implementando una política comunicacional y cultural en pos de una nueva redistribución sexual del trabajo. (Rico, 2011).

Las políticas de conciliación no han logrado una mayor equidad de género, ya que han sido pensadas principalmente desde la óptica del mercado laboral, intentando aumentar la participación laboral femenina en la medida que se faciliten a las mujeres sus responsabilidades familiares (Astelarra, 2011). Es necesario por tanto implementar políticas públicas orientadas a la modificación de la división sexual del trabajo tradicional y establecer la corresponsabilidad como principio básico de funcionamiento (Lamaute-Brisson, 2013). En el ámbito privado se deben promover cambios culturales que flexibilicen la división sexual del trabajo, mientras que en el ámbito público se debe promover el cuidado como una responsabilidad social (Batthyany, 2009). Por otra parte, resulta central en materia de conciliación visibilizar el trabajo de cuidados como una dimensión clave en los sistemas de protección social. Para ello debe discutirse acerca de los costos de implementación de servicios de cuidado, contrastándolos con los costos tanto económicos y sociales que genera su negativa a implementarlos. En este mismo sentido, la CEPAL (2012) recomienda por una parte el establecimiento de un pacto social y fiscal sobre el cuidado, cuyo financiamiento se logre a través de 
impuestos generales y por otra, la implementación de un sistema de protección social que considere al cuidado como uno de sus pilares.

\section{Políticas públicas para conciliar la vida familiar y laboral: enfoques teóricos}

\section{a) Genderfare}

Esping-Andersen (1999) afirma que la familia es tal vez el más importante fundamento social de las sociedades postindustriales. La familia no es un ámbito exclusivo de la vida privada: al jugar un rol tan preponderante en las economías en relación a la provisión de bienestar social, pasa a representar un rol clave en el análisis de las políticas públicas. El Estado debe asumir un rol más activo al considerar a las familias no sólo como sujeto de políticas públicas, sino como objeto que es posible configurar para una distribución más equitativa en relación a la participación de mujeres y hombres en los roles proveedores de bienestar social (Leiva, 2009). En el análisis debe considerarse el "contrato de género" (Esping-Andersen, 2002), en base al cual se realiza una determinada distribución de roles entre hombres y mujeres. La ciudadanía social debe considerar no solamente los estados de bienestar (welfare), sino que debe integrar las especificidades asociadas al género (gender) ${ }^{4}$. Implementando políticas públicas diferenciadas bajo la mirada del genderfare (Duncan y Pfau-Effinger, 2000) será posible alcanzar grados más amplios de inclusión social (Leiva 2009).

El cuidado de las personas dependientes puede ser asumido por diversas instituciones: el Estado, la familia, el mercado y la comunidad. Para lograr una ciudadanía social más inclusiva se hace necesario implementar políticas públicas tendientes a una desfamiliarización. Mientras la familiarización se refiere a asignar a la familia responsabilidades de provisión de bienestar social, la desfamiliarización busca aliviar a la familia en la responsabilidad de proveer servicios de bienestar social. En los países donde el Estado ha actuado desfamiliarizando a la sociedad (fundamentalmente en los países escandinavos), se ha logrado una equidad de género mayor que en los otros regímenes de bienestar (Leiva, 2009). Frente a esta discusión se plantean dos modelos: el modelo familista y el modelo desfamiliarizador (Aguirre, 2007). En el modelo familista el cuidado se torna invisible, la familia se hace cargo de los cuidados, siendo un trabajo no remunerado que realiza la mujer al interior de la familia. En el modelo desfamiliarizador el cuidado se hace visible y es posible ser medido en varios aspectos: en cuanto a sus costos y beneficios, en cuanto al uso del tiempo, en relación a la demanda del servicio, entre otros (Aguirre, 2007).

\section{b) Enfoque de derechos}

Las políticas públicas de cuidado otorgan soporte para el cuida- 
do de niños, adultos mayores, personas con discapacidad y familiares enfermos, posibilitando así conciliar la vida familiar y laboral. El sustento de las políticas públicas de cuidado es en último término el derecho que toda persona tiene a ser cuidado, enfatizando de esta manera el enfoque de derechos en materia de cuidados. Desde este enfoque se cuestiona el papel subsidiario del Estado destinado a compensar las prestaciones que no es posible obtener en el mercado de trabajo, y se promueve el papel garante de derechos. La visión de Estado subsidiario postula que el Estado debe atender a las mal llamadas mujeres vulnerables subsidiando servicios o redes comunitarias de mujeres, lo que en definitiva refuerza la división sexual del trabajo en vez de cuestionarla. Si la perspectiva de las políticas es por el contrario la titularidad del derecho, el sujeto del derecho es el niño, la niña o las personas adultas mayores que requieren cuidado (Montaño, 2010). En la misma línea argumentativa, Pautassi (2010) afirma que para algunos sectores los servicios de cuidado son prestaciones dirigidas a las mujeres. Estas posturas conservadoras afirman que se debe apoyar a las mujeres para su ingreso al mercado laboral. Sin embargo, desde el enfoque de derechos el cuidado es un derecho de todas las personas, sean infantes o adultos mayores, y debe por tanto ser garantizado a través de arreglos institucionales. Debe considerarse en el presupuesto nacional y obtener apoyo estatal.

El derecho al cuidado tiene a la igualdad y a la universalidad como principios rectores, pues se promueve que todos los ciudadanos tengan igualdad de acceso al cuidado, concebido para todas las personas como titulares de derechos (CEPAL, 2012). En efecto, Pautassi (2010) expresa que ser portador de un derecho es una pretensión jurídicamente justificada, "que habilita a una persona a hacer o no hacer algo y, a la vez, para reclamar a terceros que hagan o se abstengan de hacer algo. Es decir, existe una norma jurídica que le otorga a una persona una expectativa positiva — de acción- y una negativa —de omisión-, creando al mismo tiempo sobre otros sujetos obligaciones y deberes correlativos" (pág. 76). En este sentido se han distinguido tres tipos de derechos: los derechos civiles y políticos; los derechos socioeconómicos y culturales, y por último los derechos de desarrollo colectivo, que fueron categorizados por Karel Vasek ya en 1977 como derechos de primera, de segunda y de tercera generación (Callaway y Harrelson-Stephens, 2007). Los derechos de tercera generación están vinculados al principio de solidaridad e incluyen el derecho a la paz, al desarrollo, a un ambiente saludable, entre otros.

\section{El programa IGUALA: Características y evaluación gubernamental}

La necesidad de establecer criterios de equidad en la administración del Estado durante el primer Gobierno de Bachelet impulsó diver- 
sas políticas sociales y programas de Estado. La discriminación de la mujer respecto al acceso a trabajos remunerados, las brechas salariales frente a la realización de labores iguales o equivalentes a las que efectúan los hombres y los temas de cuidado en el hogar que, por considerarse una responsabilidad femenina, limitan su entrada al mercado laboral, fueron la base del Código de Buenas Prácticas Laborales. Sus objetivos apuntan a garantizar condiciones que promuevan la igualdad de oportunidades, asegurando los principios de no discriminación e igualdad de trato entre mujeres y hombres. El código definió directrices en siete dimensiones que permiten medir el cumplimiento del principio de Igualdad: Proceso de reclutamiento- selección, desarrollo de carrera y acceso a capacitación, representación equilibrada o paritaria entre mujeres y hombres en cargos de jefatura y de responsabilidad directiva, condiciones de trabajo, protección de los derechos de maternidad y responsabilidades parentales, conciliación de responsabilidades laborales con obligaciones familiares y prevención y sanción del acoso laboral y/o sexual en el trabajo.

Si bien se incluyeron sus propuestas en la gestión de servicios públicos, quedaba aún como desafío la implementación de estas prácticas en el área privada. Es así como nace en el año 2008 el Programa IGUALA de SERNAM, cuyo objeto es la instalación del principio de igualdad en el mundo laboral privado. El Código de Buenas Prácticas Laborales se constituye en el principal insumo del Programa IGUALA, el cual considerando las siete dimensiones mencionadas permite medir cómo se comportan las mismas en las empresas que se adscriben voluntariamente al programa. Si la compañía cumple adecuadamente con la implementación del programa, el SERNAM le ofrece la certificación de calidad del sello IGUALA, que se obtiene luego de un proceso de dos años. ${ }^{5}$

Para implementar el programa las empresas adscritas a éste proceden a realizar un autodiagnóstico de la situación inicial, en el que participa por un lado el SERNAM y por otro la contraparte de la empresa, estableciendo un Plan de Acción con remediales y nuevas propuestas surgidas desde la compañía. Este plan se monitoriza posteriormente con reuniones de coordinación y revisión de reportes.

En el año 2010 el SERNAM realiza la primera evaluación del programa desde el año del inicio en 2006 al 2009 (Orellana, 2010). Los resultados e indicadores de la evaluación-sistematización del programa se basan principalmente en información entregada por los organismos públicos y las empresas. Sobre la calidad, la información considera encuestas aplicadas a trabajadores que evalúan sin embargo sólo su satisfacción en cuanto a la asesoría entregada por SERNAM al organismo público o empresa. Además, evalúa los cambios en las prácticas laborales, pero no indica cuáles son estas prácticas. Por otra parte, los indicadores que dicen relación con las acciones de difusión del programa para con los beneficiarios no son evaluados en el porcentaje de 
logro. De esta manera se evidencia que no se realiza la evaluación desde la percepción del beneficiario en cuanto a la pertinencia de las medidas y a cómo esto ha implicado cambios en su entorno familiar.

Llama la atención que en la evaluación efectuada, la calidad es entendida como la satisfacción de las empresas con la asesoría entregada por el SERNAM. Debería por tanto reemplazarse este indicador por otro centrado en el impacto en la población beneficiada, lo que implica una evaluación empírica de la política por parte de las personas e instituciones beneficiadas. Otro aspecto que llama la atención es la dificultad de establecer en el sector privado los beneficiarios reales del programa, ya que se consideran como beneficiarios tanto empresas, instituciones y personas, haciendo difícil la diferenciación de resultados en la evaluación. Parece, por otra parte, inconveniente que muchos de los resultados sean recogidos a través de informes de las empresas u organismos y no mediante preguntas a trabajadores y trabajadoras. Además, el instrumento de recolección de datos en algunas ocasiones fue una encuesta, la que no da lugar suficiente para una profundización de las opiniones.

La investigación que aquí presentamos da un paso más allá a estas limitaciones. En primer lugar utiliza una metodología cualitativa aplicación de entrevistas en profundidad en todos los niveles considerados- lo que posibilita a las personas explayarse en sus observaciones sobre cómo han recibido el impacto de las medidas implementadas. En segundo lugar se realiza la evaluación enfocándose en la percepción de los trabajadores y trabajadoras. En tercer lugar indaga específicamente en el logro de la conciliación entre la vida familiar y laboral, resaltando aciertos y dificultades de las medidas.

\section{Evaluación de las medidas implementadas para conciliar la vida familiar y laboral del Programa IGUALA en una compañía minera en la región de Tarapacá}

En este apartado se analiza cómo fueron implementadas las medidas tendientes a lograr la conciliación entre la vida familiar y laboral en una empresa minera en Tarapacá, destacando los aciertos y obstáculos presentados en el proceso. ${ }^{6}$ Se seleccionó una compañía minera para realizar el estudio porque fue una empresa premiada a nivel nacional durante el primer año de aplicación del programa. Se entrevistaron a dos informantes claves del SERNAM de la región de Tarapacá, ubicado en la ciudad de Iquique. Uno de ellos coordinó la implementación del programa al comienzo, y el segundo se hizo cargo posteriormente de ella. En la compañía minera en estudio, se entrevistó al informante clave que le correspondió implementar el programa. Se entrevistó además a cuatro beneficiarios/as del programa, dos hombres y dos mujeres, correspon- 
diendo a trabajadores contratados directamente por la compañía y que tenían hijos menores de seis años.

Según expresamos anteriormente, el programa considera siete dimensiones. Cada dimensión contempla tres parámetros a evaluar: en primer lugar las acciones de información que ha implementado la empresa para informar o difundir sobre las adecuadas prácticas laborales a sus empleados; en segundo lugar las acciones y políticas concretas que se han desarrollado en el ámbito (refiriéndose a aquellas que deberían constatarse según el marco legal chileno) y por último las acciones positivas que se traducen en parámetros para evaluar medidas o políticas implementadas por la empresa que sobrepasen las exigencias legales nacionales. En el programa IGUALA la conciliación de responsabilidades laborales con obligaciones familiares corresponde a una dimensión, en tanto que la protección a los derechos de maternidad y responsabilidades familiares parentales se establece en otra dimensión. Como esta última materia comprende acciones relacionadas con el área de los cuidados de terceros que influyen en la conciliación, nuestro estudio abarca el análisis de ambas dimensiones (ver tablas 1 y 2).

A continuación se analizan las medidas para conciliar la vida familiar y laboral del programa en tres niveles: en el primero se realiza una reflexión sobre las medidas considerando los dos marcos teóricos propuestos, en el segundo se analizan los obstáculos de la compañía minera para implementar las acciones, y por último se examinan las opiniones de los beneficiarios del programa.

\section{a) Genderfare y enfoque de derechos en las medidas de conciliación}

Las medidas del programa IGUALA tendientes a fomentar la conciliación entre vida familiar y laboral no han sido concebidas desde un régimen de genderfare ni desde un enfoque de derechos. No se consideró el genderfare (Duncan y Pfau-Effinger, 2000) en su diseño, ya que sus objetivos apuntaron al crecimiento económico mediante la inclusión laboral de la mujer y a un cambio cultural en la igualdad de género, sin embargo no se contemplaron medidas para que el Estado asuma una corresponsabilidad en las tareas de cuidado. De esta manera, el programa no tiende a la desfamiliarización (Aguirre, 2007), sino que supone que la familia es la responsable principal del cuidado. Tampoco aplicó el programa un enfoque de derechos pues no apunta a una comprensión del cuidado como un derecho de todas las personas que lo requieran (Montaño, 2010; Pautassi, 2010; Calderón, 2013), sino como un beneficio a las mujeres. La gran deuda del programa IGUALA es por una parte hacer efectiva una política que permita fomentar la corresponsabilidad del Estado con miras a una desfamiliarización y por otra parte enseñar a muy temprana edad que niños y niñas, adultos mayores y personas con discapacidad son titulares del derecho a ser cuidado, y que en tanto derecho, el Estado tiene el deber de garantizarlo. 


\section{b) Obstáculos en la implementación de las medidas para conciliar la vida familiar y laboral}

La implementación de las medidas para conciliar la vida familiar y laboral llevada a cabo por la empresa minera -considerando tanto los indicadores propuestos en la dimensión de conciliación y la dimensión de protección a la maternidad del programa- presentó una serie de obstáculos. Las dificultades se observan principalmente en tres ámbitos: motivación para adherirse al programa, acciones que la compañía implementó, pero que no representan un real avance, y por último, medidas que no pudieron implementarse debido a la naturaleza del sistema de turnos de la minería. A continuación se analizarán estas dificultades.

\section{b1 Motivación de la empresa para participar en el programa}

Existió una dificultad de motivar a las empresas a participar en el programa, por lo que sólo participaron primero tres empresas a nivel regional: dos del sector extractivo y una del sector servicios de salud, uniéndose posteriormente una empresa comercial. De acuerdo a la visión de los informantes claves del SERNAM Regional, las empresas generalmente no visualizan el aporte que la implementación de medidas de conciliación y buenas prácticas laborales tiene para ellas. La adscripción al programa se traduce en la aspiración de obtener el sello IGUALA para ser considerada como una empresa de altos estándares de gestión, anteponiendo este objetivo al de la no discriminación laboral. Por su parte, según el informante clave de la empresa minera, la decisión de participar y adscribirse voluntariamente al programa no fue de complejidad para la compañía, porque ésta presenta estándares internacionales en sus políticas de gestión de Recursos Humanos. En razón de ser una empresa transnacional, cuenta con capitales y directrices de la casa matriz en el extranjero, que resultan superiores al común de las empresas nacionales. La mayoría de las medidas solicitadas en las siete dimensiones, de acuerdo a su percepción, no fueron difíciles de cumplir, porque eran medidas que ya existían previamente como parte de las políticas de la compañía y no tuvieron que diseñarse especialmente para el programa. En efecto, así lo expresa el entrevistado:

“...nosotros voluntariamente ya las habíamos tomado como algo de la compañía, entonces yo creo que la motivación es como trabajar para seguir en el mismo rumbo que ya se había tomado en el fondo, como por ejemplo la igualdad salarial eso ya estaba, eso no fue una consecuencia del convenio, porque ya como política para Minera Norte $^{7}$ la igualdad salarial estaba ya implementada" (entrevista a contraparte de la compañía minera).

Del análisis de las medidas implementadas para conciliar la vida familiar y laboral pudo observarse que no tendieron hacia un cambio de la empresa minera en esta dirección, sino que la motivación estuvo dada 
porque la compañía tenía en gran parte implementadas las medidas y podía así optar fácilmente al sello IGUALA.

\section{b 2 Acciones implementadas: ¿¿real avance?}

Las acciones para conciliar la vida familiar y laboral implementadas por el programa son en su mayoría acciones informativas. Además, el programa considera las acciones y políticas que se amparan en el marco legal vigente y las acciones positivas, es decir, aquéllas que van más allá de la ley. Las tablas 2 y 3 ilustran estas situaciones. En ellas se muestran las dimensiones de conciliación de responsabilidades familiares con obligaciones laborales y de protección de los derechos de maternidad y responsabilidades parentales, así como las medidas específicas implementadas por la empresa. Las acciones informativas implementadas, en ambas dimensiones, utilizan principalmente cuatro medios: las boletas informativas, las publicaciones en una revista interna, la emisión de folletos y la difusión mediante actividades recreativas en familia. El Programa Comunicacional Buenas Prácticas Laborales se realiza a través de boletas informativas, son especies de gigantografías que se ubican en lugares visibles, como a la salida del casino. A través de la página web no es posible difundir información, pues éstas se manejan de forma centralizada desde la casa matriz de la compañía.

En relación a las acciones y políticas que implementó la empresa minera que están dentro del marco legal vigente, así como aquellas que sobrepasan la ley, debe destacarse que la compañía no está autorizada a reformular las políticas. Por tratarse de una empresa internacional, el informante clave indica que hay ciertas medidas que no pueden implementarse, pues ellos como filial no están autorizados a realizar cambios a las políticas de la empresa, sino que tales modificaciones deben provenir de la casa matriz ubicada en el extranjero. Para subsanar esto y cumplir así con la implementación del programa- la compañía minera utiliza el recurso de declarar que la empresa tiene ya una política a este respecto, y que tiene implementado un reglamento correspondiente. Dentro de las acciones y políticas que están de acuerdo con el marco nacional, fue implementada una de la dimensión de conciliación. La acción referida a revisar y cambiar la política de capacitación de la empresa, buscando con ello organizar los tiempos de las capacitaciones en horario laboral, se cumple a través de un contrato colectivo que la compañía ya tiene implementado. Con respecto a las acciones positivas, es decir, aquellas que van más allá de lo solicitado por la ley, hubo varias medidas que del mismo modo ya existían en la empresa y estaban consignadas en sus reglamentos. En la dimensión de conciliación, la acción destinada a entregar apoyo económico a trabajadores/as con infantes menores de dos años se cumple a través de un bono de sala cuna. La acción referida a entregar apoyo económico a trabajadores/as que tienen personas dependientes con alguna inhabilidad física o mental se cumple a través de un contrato colectivo de trabajo y por medio de un seguro complementario de salud. En la dimensión de protección a la 
maternidad se cumplieron tres de las seis acciones positivas sugeridas. Con respecto a la acción positiva de ampliar el periodo de alimentación más allá de lo legal (una hora), la compañía dispone que las mujeres en periodo de lactancia no deben trabajar en faena sino en la casa matriz ubicada en Iquique. Además, afirma el entrevistado que no hay un control estricto si la madre se toma más de la hora que le corresponde legalmente, ya que no se marca tarjeta por este derecho. Sobre la acción positiva de reorganizar los horarios de las trabajadoras en estado avanzado de embarazo, la compañía cuenta con un reglamento que dispone que las trabajadoras embarazadas deben ser trasladadas de la faena a la casa matriz. Sobre la acción positiva buscar los mecanismos necesarios para dar cumplimiento a los derechos maternales y responsabilidades parentales, la empresa cuenta con un reglamento que consigna el pago de un bono a la madre. Este bono puede ser utilizado para pagar una sala cuna o servicio doméstico de cuidado de niños.

La implementación de las medidas informativas para conciliar la vida familiar y laboral, si bien es importante, ello no tiene el mismo efecto que impulsar una acción concreta. Asimismo lo entiende el propio programa, que otorga una valoración sólo de un décimo a la acción informativa de protección a los derechos de maternidad, frente a los nueve décimos que se consideran para las otras acciones. De la misma manera, en la dimensión de conciliación del programa, las acciones informativas puntúan sólo un cuarto, frente a los tres cuartos que se otorgan a las acciones concretas.

Con respecto a la implementación de las acciones dentro del marco legal y las acciones que van más allá de la ley, pudo constatarse que una cantidad importante de las medidas no son fruto del programa, sino que corresponden a medidas que la compañía ya tiene incorporadas como parte de sus políticas internas, sean políticas de recursos humanos, de capacitación, o similares. En la última columna de las tablas 2 y 3 se indica si esta medida fue implementada para cumplir el programa o si por el contrario no es fruto del programa, sino que existía desde antes como una política de la compañía minera. En este sentido, la forma en que la empresa minera implementó las medidas de conciliación del programa IGUALA se acercan más a la realización de un diagnóstico de la situación antes que al desarrollo de una política pública, lo que hace pensar que en materia de conciliar vida familiar y laboral no se produjo un real avance. 


\section{Tabla 1 Dimensión Conciliación de responsabilidades laborales con obligaciones familiares}

\begin{tabular}{|c|c|c|c|}
\hline $\begin{array}{l}\text { Categona de } \\
\text { acciones }\end{array}$ & Acciones propuestas por el SERNAN & $\begin{array}{l}\text { Acciones implementadas por la } \\
\text { compañia minera }\end{array}$ & $\begin{array}{l}\text { Accion } \\
\text { implementa } \\
\text { da a raiz del } \\
\text { programa }\end{array}$ \\
\hline \multirow[t]{7}{*}{ Informacion } & \multirow{4}{*}{$\begin{array}{l}\text { T Difundir por todos los medios que } \\
\text { disponga la empresa sobre la importancia } \\
\text { del desarrollo de horabres y mujeres en su } \\
\text { integralidad como trabajadoras-es, padres y } \\
\text { personas. }\end{array}$} & $\begin{array}{l}\text { Programa Comunicacional Buenas } \\
\text { Practicas Laborales }\end{array}$ & S1 \\
\hline & & Reportaje l en revista intema & S1 \\
\hline & & Reportaje 2 en revista intema & S1 \\
\hline & & $\begin{array}{l}\text { Difusion de actividades de recreacion } \\
\text { para la familia }\end{array}$ & S1 \\
\hline & \multirow{3}{*}{$\begin{array}{l}2 \text { Difundir por todos los medios que } \\
\text { disponga la empresa la importancia de la } \\
\text { corresponsabilidad ente mujeres y hombres } \\
\text { respecto del hogar y sus hijas o hijos. }\end{array}$} & $\begin{array}{l}\text { Programa Comunicacional Buenas } \\
\text { Practicas Laborales }\end{array}$ & \$1 \\
\hline & & Reportaje 3 en revista intema & $\$ 1$ \\
\hline & & Difusion de actividades en familia & $\$ 1$ \\
\hline \multirow[t]{3}{*}{$\begin{array}{l}\text { Acciones y/0 } \\
\text { politicas }\end{array}$} & $\begin{array}{l}\text { I Revisar y cambiar, si corresponde, la } \\
\text { politica de recursos humanos que promueve } \\
\text { la conciliacion de responsabilidades } \\
\text { laborales con obligaciones familiares. }\end{array}$ & No aplica & \\
\hline & $\begin{array}{l}2 \text { Revisar y cambiar, si corresponde, la } \\
\text { politica de capacitacion de la empresa, } \\
\text { buscando conello organizar los tiempos de } \\
\text { las capacitaciones en horario laboral. }\end{array}$ & Contrato Colectivo & No \\
\hline & $\begin{array}{l}3 \text { Revisar y cambiar. si corresponde. las } \\
\text { jomadas laborales de las y los trabajadores } \\
\text { que desean conciliar su vida familiar con la } \\
\text { laboral. flexibilizando y mejorando su } \\
\text { jomada. }\end{array}$ & No aplica & \\
\hline \multirow[t]{6}{*}{$\begin{array}{l}\text { Acciones } \\
\text { positivas }\end{array}$} & $\begin{array}{l}\text { Entregar apoyo economico a los y las } \\
\text { trabajadores con hijos e hijas menores de } \\
\text { dos anos. independientemente del sexo del } \\
\text { trabajador(a). }\end{array}$ & Bono sala cuna (anexo contrato) & No \\
\hline & \multirow{2}{*}{$\begin{array}{l}2 \text { Entregar apoyo economico a los y las } \\
\text { trabajadores que tienen personas } \\
\text { dependientes con alguna inhabilidad fisica o } \\
\text { mental. }\end{array}$} & Contrato colectivo de trabajo & \multirow[t]{2}{*}{ No } \\
\hline & & $\begin{array}{l}\text { Seguro complemento de salud } \\
\text { (contrato colectivo de trabajo) }\end{array}$ & \\
\hline & $\begin{array}{l}3 \text { Habilitar para los hijos de los y las } \\
\text { trabajadores el servicio de guarderia y/o } \\
\text { cuidado posterior del colegio jardin infantil. }\end{array}$ & No aplica & \\
\hline & $\begin{array}{l}4 \text { Habilitar para los hijos de los y las } \\
\text { trabajadores el servicio de tutoria en } \\
\text { materias escolares. }\end{array}$ & No aplica & \\
\hline & $\begin{array}{l}5 \text { Habilitar para los hijos de los y las } \\
\text { trabajadores el servicio de guarderia y } \\
\text { atencion en periodos de vacaciones } \\
\text { escolares y diasno lectivos durante el año. }\end{array}$ & No aplica & \\
\hline
\end{tabular}

Fuente: Elaboración propia en base a información entregada por el SERNAM y por la empresa minera estudiada. 
Polis, Revista Latinoamericana, Volumen 14, No 42, 2015

\section{Tabla 2 Dimensión Protección de los derechos de maternidad y responsabilidades parentales}

\begin{tabular}{|c|c|c|c|}
\hline $\begin{array}{l}\text { Categona de } \\
\text { acciones }\end{array}$ & Acciones & $\begin{array}{l}\text { Acciones implementadas por la } \\
\text { companila minera }\end{array}$ & $\begin{array}{l}\text { Accion } \\
\text { implementa } \\
\text { da a raiz del } \\
\text { programa }\end{array}$ \\
\hline \multirow[t]{2}{*}{ Informacion } & \multirow{2}{*}{$\begin{array}{l}\text { 1 Difundir por todos los medios que } \\
\text { disponga la empresala legislacion vigente } \\
\text { que protege la matemidad y promueve } \\
\text { responsabilidades parentales, a todos/as los } \\
\text { trabajadores/as independientemente de su } \\
\text { condicion contractual. }\end{array}$} & $\begin{array}{l}\text { Programa Corrunicacional Buenas } \\
\text { Practicas I aborales }\end{array}$ & S1 \\
\hline & & $\begin{array}{l}\text { Regamento Intemo de Orden, } \\
\text { Higiene y Seguridad }\end{array}$ & No \\
\hline $\begin{array}{l}\text { Acciones y/o } \\
\text { politicas }\end{array}$ & $\begin{array}{l}\text { I Revisar y reformular la politica de } \\
\text { recursos mumos - si corresponde - que } \\
\text { promueva el uo delos instrumentos legalas } \\
\text { vigentes queprotegen la matemidad y las } \\
\text { responsabilidades parentales. a todos/as los } \\
\text { trabajadores/a independientemente de su } \\
\text { condicion contractual. }\end{array}$ & $\begin{array}{l}\text { Programa Comunicacional Buenas } \\
\text { Practicas Laborales }\end{array}$ & S1 \\
\hline \multirow[t]{7}{*}{$\begin{array}{l}\text { Acciones } \\
\text { positivas }\end{array}$} & $\begin{array}{l}\text { I Amplia el penodo dealimentacion y } 10 \text { no } \\
\text { considerar en el tiempo queindica la Ley, el } \\
\text { tiempo de traslado de las mujeres } \\
\text { trabajadoras independientemente de su } \\
\text { condicion contractual. }\end{array}$ & $\begin{array}{l}\text { Tndice procedimiento de proteccion a } \\
\text { la matemidad }\end{array}$ & No \\
\hline & $\begin{array}{l}2 \text { Disenar e implementar acciones que } \\
\text { permitan que los padres puedan asumir sus } \\
\text { responsabilidades antes, en el momento y } \\
\text { posterior al nacimiento de una hija o un } \\
\text { hijo. independientemente de su condicion } \\
\text { contractual. }\end{array}$ & No aplica & \\
\hline & $\begin{array}{l}3 \text { Reorganizar los horarios de las } \\
\text { trabajadoras en el caso de encontrarse en } \\
\text { estado avanzado de embarazo. } \\
\text { independientemente de su condicion } \\
\text { contractual. }\end{array}$ & Anexo de contrato bono sala cuna & No \\
\hline & $\begin{array}{l}4 \text { Ampliar el post natal masculino en al } \\
\text { menos un } 50 \% \text { de bestablecido por la ley. } \\
\text { de los trabajadore independientemente de } \\
\text { su condicion contractual. }\end{array}$ & No aplica & \\
\hline & $\begin{array}{l}5 \text { permitir a los hombres con hifos-as recian } \\
\text { nacidos optar por jomadas de trabajo } \\
\text { flexibles. con los mismos derechos y } \\
\text { beneficios que tenia con anterioridad al } \\
\text { nacimiento de su hijo/a, } \\
\text { independientemente de su condicion } \\
\text { contractual. }\end{array}$ & No aplica & \\
\hline & $\begin{array}{l}\text { o Buscar los mecanismos necesarios para } \\
\text { dar cumplimiento a los derechos matemalx } \\
\text { y responsabilidades parentales. } \\
\text { independientemente de su condición } \\
\text { contractual en caso que el trabajo propio de } \\
\text { la empresa involucre jomadas } \\
\text { excepcionales o sistemas de tumos. }\end{array}$ & Anexo de contrato bono sala cuna & No \\
\hline & COMODIN & $\begin{array}{l}\text { Procediniento proteccion a la } \\
\text { matemidad }\end{array}$ & No \\
\hline
\end{tabular}

Fuente: Elaboración propia en base a información entregada por el SERNAM y por la empresa minera estudiada.

\section{b 3 Medidas que por la naturaleza de la compañía no son aplicables}

Otra dificultad para implementar las medidas de conciliación por parte de la compañía minera es que muchas veces los indicadores de las dimen- 
siones diseñadas por el organismo estatal no coinciden con la realidad de la empresa. Por tratarse de una faena en altura retirada de la ciudad, los turnos, por ejemplo de siete días internos en los campamentos mineros y siete días de descanso en el hogar, hacen impracticable implementar ciertas medidas. En estos casos la empresa determinó que la medida no era aplicable. En las tablas 1 y 2 puede observarse que existen cinco acciones en la dimensión de conciliación, y tres en la dimensión de protección de los derechos de maternidad que la empresa determina que "no aplica".

Para subsanar de alguna forma que varias acciones positivas eran inaplicables, la empresa decidió utilizar un “comodín” que dice relación con varios aspectos de protección a la maternidad distintos a los referidos, los que estaban ya consignados en las políticas de la empresa. De acuerdo a lo expresado por los informantes claves del SERNAM Regional, en algunos casos las empresas pueden reemplazar una medida por otra, a la que han llamado “comodín”, facilitándoles así el cumplimiento del programa y la obtención del sello IGUALA.

\section{c) Percepción de los beneficiarios/as del programa IGUALA}

Los análisis de los relatos de las dos mujeres y dos hombres entrevistados se estructuraron en torno a dos ejes: los beneficios de parentalidad y las medidas de conciliación entre vida familiar y laboral.

\section{c1 Beneficios de parentalidad}

La percepción de los beneficios laborales en general es altamente positiva, contando además con bonos escolares y de salud. Si se tiene en cuenta que las remuneraciones en las compañías mineras son más altas que el promedio, no es de extrañar que una trabajadora exprese al respecto:

“En la parte de las remuneraciones y bonos, efectivamente tenemos nosotros un apoyo en la parte escolar, además tenemos buen seguro de salud y la verdad es que en la parte remuneración es una rebuena opción, porque a veces las mamás que somos solas tenemos que esforzarnos un poco más pa' lograr algunas cosas y a mí me complace en el fondo lo que yo busco." (Andrea, 35 años. Asistente de producción. Hija de 5 años).

Se observa también satisfacción por los beneficios que la empresa entrega por el nacimiento de un hijo, además del monto de dinero proporcionado por la caja de compensación. Sobre esto un trabajador afirma:

“...sí, hay un pago por el nacimiento de hijos, la empresa te da... fueron como $\$ 60.000$, aparte de eso también, la caja de compensación, que es la caja que está afiliada de Minera Norte, también te da plata, son $\$ 22.000 \ldots$ A través de recursos humanos, yo hice las consultas previas, y ahí me dijeron todos los derechos, los benefi- 
cios de paternidad...” (Pablo, 34 años. Cargo operativo, mecánico hidráulico de planta. Tres hijos, de 13 y 7 años y de 6 meses).

En cuanto a los beneficios correspondientes a la maternidad, la compañía minera decide trasladar a una operaria de máquinas desde las faenas localizadas en altura a las oficinas ubicadas en la ciudad al enterarse de que está embarazada. Esta acción puede catalogarse como una acción positiva pues va más allá de lo que la ley establece. El espíritu que mueve esta acción en la compañía está dado por otorgar un grado mayor de seguridad a la futura madre en tanto se minimizan los riesgos de encontrarse durante el embarazo físicamente muy alejada de un hospital o centro de salud.

“...dije qué va a pasar conmigo ahora, porque como trabajaba en maquinaria pesada, dije a lo mejor se me puede hacer complicado, entonces conversé con Recursos Humanos y me dieron solución al tiro, no tuvieron drama, ellos sí igual quedaron impactados en primer momento, porque era primera vez que una operadora en minas quedaba embarazada, entonces... pero no, no hubo ningún problema, eh...ellos me gestionaron para poder bajar y trabajar en la administración, ahí estuve cuando empezó el prenatal” (Fernanda, 25 años. Operadora de maquinaria pesada. Hijo de dos años y medio).

Por otra parte, luego del alumbramiento, a la madre se la mantiene ubicada en los centros administrativos ubicados en la ciudad. Al principio la entrevistada piensa que el cambio de localización desde las faenas en altura al centro administrativo en la ciudad es un beneficio que le entrega la compañía minera, sin embargo luego reconoce que por el rubro es la forma de hacer cumplir lo que legalmente está establecido: otorgar el tiempo de una hora para el amamantamiento del recién nacido.

(...) Lo que pasa es que hay como una ley parece cierto? que uno tiene que ver al hijo una hora producto del amamantamiento y todo eso, entonces por eso no me podían subir a la mina, porque era algo arriesgado no sé, porque una de dos, cómo yo iba a bajar por una hora o subir mi bebé por una hora allá, era bastante complicado, entonces por eso dijeron ya mejor te quedas en la administración...” (Fernanda, 25 años. Operadora de maquinaria pesada. Hijo de dos años y medio).

\section{c2 Medidas de conciliación entre vida familiar y laboral}

En cuanto a los permisos en caso de alguna enfermedad de un hijo o familiar, los entrevistados manifiestan que éstos se otorgan de acuerdo al criterio de los supervisores directos, siendo un aspecto que a veces no está regulado mediante un beneficio formal. De hecho, ante la situación del nacimiento ad portas de un hijo, uno de los trabajadores expresa:

“.... Me dieron unos días, pero, acá hay un tema, que de cierto modo, 
la compañía favorece este tipo de actitudes (pago de bonos, permisos, etc.). En las mismas jefaturas nacen este tipo de iniciativas, como Hueón, vai’ a tener un hijo, tú le avisas a tu líder, a tu supervisor que vas a tener un hijo, cachai? que a lo mejor lo vas a tener, entonces por un tema de riesgo de tenerte a ti pensando en la mina a kilómetros por tu hijo, muchas veces ellos te dejan ir, y te dicen: no, tómate tu tiempo, estáte tranquilo, y cuando puedas subir y estar bien, tú subes, entendí?...”. (Alejandro, 29 años. Ingeniero metalúrgico. Hijo de 3 años).

El criterio del supervisor es altamente valorado por los trabajadores, quienes ante la eventualidad de necesitar un permiso de manera urgente, sienten que pueden contar con la venia de sus superiores.

“...Sí, hay permisos, pero uno tiene que conversar con el supervisor y llegar a un trato poh, yo se los puedo devolver, o me puedo tomar días de vacaciones...” (Pablo, 34 años. Cargo operativo, mecánico hidráulico de planta. Tres hijos de 13 y 7 años y de 6 meses).

Por otra parte, al ser un beneficio no formalizado, muchas veces el cuidado de los hijos enfermos es asumido por otras mujeres en el hogar del trabajador (a) (actúa bajo lógica de familiarización).

“eh... a mí en algunas oportunidades se me hizo complicado sí, cuando se me enfermaba el bebé, pero tenía a mi mami, ella lo cuidaba, entonces.... pero a veces cuando tenía que verlo yo obviamente pedía permiso (Fernanda, 25 años. Operadora de maquinaria pesada. Hijo de dos años y medio).

Un resultado emergente de esta investigación es el efecto que el sistema de turnos tiene en la dinámica de la familia y el cumplimiento de roles. Un trabajador nos expresa que el sistema de trabajo de la minería favorece una mayor frecuencia de separaciones matrimoniales. El sistema de turnos resulta en extremo agotador para la dinámica familiar, pues se establecen dos rutinas muy distintas, una cuando el trabajador está en casa, y otra cuando se encuentra en su lugar de labores. Esta doble rutina es explicada del siguiente modo por un entrevistado:

“...yo en un tiempo nos separamos, porque es súper fuerte los cambios de turno, es fuerte, fuerte.....imagínate tú trabajas en una mina, dónde tú trabajas, después te vas a almorzar, a cenar, tu pieza, estás solo como en un hotel y después llegai a la casa y empiezas a notar cosas, ya no puedes tirar las cosas al suelo, tuviste cuatro días, pero ahora tenís que cumplir ciertas normas, entonces de un mundo sin normas a un mundo con normas, hay choque, a lo mejor tú en la mina no te gusta que dejen el celular arriba de la tele, pero no sé cómo lo podría favorecer la compañía, para que se ayudara a manejar situaciones de inserción al descanso, más en los siete por siete y los viejos pasan siete días solos y siete días con los niños, y los niños 
no lo aguantan, cachai, entonces eso genera choque, entonces mucha gente se separa” (Alejandro, 29 años. Ingeniero metalúrgico. Hijo de 3 años).

Como consecuencia de esta dificultad para conciliar la vida familiar y laboral, el entrevistado optó por cambiarse de compañía minera para trabajar en las dependencias administrativas ubicadas en la ciudad, obviando así los sistemas de turnos.

"Mira, es bien rara, yo trabajaba en Minería Sur cuando Álvaro estaba bien chico, y me tocaba irme los domingos a las doce de la noche, y llegar a las seis de la mañana y tomar otro bus y llegar a las diez a la mina y a 3500 metros. Y el jueves, me tocaba tomar un bus a las seis de la mina, bajar dos horas, después tomar un bus a Iquique, cuatro de la mañana, muerto, así me despegaban del asiento jja ja ja! y el viernes era trámite en la mañana, en la tarde era dormir, y el sábado y domingo yo forzaba dormir más, entonces mi relación con Álvaro era “ya juguemos un ratito, ya ya, pero déjame dormir" entendí, era como súper lejana, él tampoco en cierto modo me hacía mucho caso, se crió con la mamá, entonces como que la mamá era como el pilar de él, la autoridad, y yo pasaba peleando con él, tratar de imponer mi autoridad. Y cuando me cambié de pega, y me cambié acá por eso mismo lo hice, para trabajar en la ciudad...” (Alejandro, 29 años. Ingeniero metalúrgico. Hijo de 3 años).

Sin embargo, el sistema de turnos no incomoda a todas las personas. Algunas declaran que este sistema es mejor para la vida familiar, pues posibilita pasar más tiempo con sus hijos. A este respecto se entrega el relato de una trabajadora:

“estoy tres días en la casa, tres días efectivos donde son súper intensos con tu hijo, uno está intensamente aprovechando el tiempo. Porque por ejemplo cuando yo trabajaba en ciudad, yo llegaba todos los días, pero llegaba a dormir a la casa, y ella también estaba durmiendo...” (Andrea, 35 años. Asistente de producción. Hija de 5 años).

\section{Conclusiones y reflexiones finales}

El objetivo de este artículo se centró en indagar cómo fueron implementadas las medidas tendientes a conciliar la vida familiar y laboral en una empresa minera en la región de Tarapacá, que el programa IGUALA contempló. Los resultados obtenido s evidencian que el programa pretende instalar buenas prácticas laborales a través de la implementación de una serie de acciones relacionadas al Trabajo Decente. Sus objetivos responden por un lado a reducir las brechas de participación de las mujeres en el mercado laboral para aumentar el crecimiento económico y por otra parte a impactar en un cambio cultural de equidad de género en el trabajo. 
Al analizar las medidas para conciliar la vida familiar y laboral y contrastarlas con las perspectivas teóricas de genderfare (Duncan y PfauEffinger, 2000) y enfoque de derechos (Pautassi, 2010), necesarias para lograr éxito en la implementación de las acciones, se concluye que ninguna de estas perspectivas está presente. En efecto, se aprecia que la corresponsabilidad estatal no ha sido considerada (OIT, 2009) y que el programa no tiende a una desfamiliarización (Aguirre, 2007), desaprovechando la oportunidad de que el Estado participe en el bienestar social considerando la perspectiva de género. Por otra parte, no se integra tampoco el enfoque de derechos (Montaño, 2010) en las acciones para conciliar familia-trabajo, invisibilizando que las personas receptoras de cuidado tienen el derecho a ser cuidadas con independencia de la participación laboral de la mujer. Así, las medidas consideradas en el programa tendientes a conciliar la vida familiar y laboral parecen responder a una visión conservadora en tanto buscan apoyar a las mujeres para lograr su ingreso al mercado laboral.

Los resultados del estudio mostraron además que la compañía minera enfrentó una serie de dificultades al implementar las medidas para conciliar la vida familiar y laboral. El programa consideró la adopción de tres tipos de medidas: acciones informativas, acciones que están dentro del marco legal vigente y acciones que van más allá de la ley. La empresa implementó en su mayoría acciones informativas, las que sin embargo tienen una importancia menor, tal como lo reconoce el programa al otorgar una puntuación más baja a este tipo de acciones. Sobre la adopción de los otros tipos de medidas, se destaca que la compañía carece de autonomía para llevar adelante cambios sustantivos, ya que al ser una filial de una industria extranjera no está autorizada a implementar políticas. La estrategia utilizada por la empresa consistió en dar por aplicada la acción entregando antecedentes de los reglamentos que la firma ya tenía implementados.

Otra dificultad exhibida por el programa se refiere a que varias medidas resultaron ser inaplicables en razón de la naturaleza de las faenas mineras. En las compañías mineras las obras se realizan en sistemas especiales de turnos (siete días de trabajo y siete de descanso, cuatro días de trabajo y tres de descanso, etc.), por lo que no son aplicables acciones relativas a flexibilizar la jornada de trabajo. De esta manera, hubo varias medidas que no pudieron implementarse y que la empresa declaró como inaplicables.

Es importante destacar que la motivación de la compañía minera para adherir al programa no necesariamente es buscar la equidad de género de sus trabajadores. La empresa en estudio posee ya altos estándares en esta materia, entonces al aplicar este programa no busca implementar medidas que tiendan a este fin, sino más bien obtener la certificación del sello IGUALA, lo que le permitirá destacar su gestión como empresa a nivel nacional. Esta situación permite poner de relieve que al aplicar una política pública los objetivos no necesariamente son los mismos para los diversos actores, lo que nos lleva a resaltar la importancia de tener en cuenta la diversidad de 
motivaciones de los actores involucrados a fin de lograr que efectivamente éstos se interesen en llevar adelante una política pública.

Otro aspecto a destacar dice relación con las acciones del programa dirigidas a todos los trabajadores independientemente de su condición contractual. En esta materia téngase presente que en las compañías mineras existe un alto porcentaje de trabajadores subcontratados (Leiva y Campos, 2013; Leiva, 2012) ${ }^{8}$. Al no ser trabajadores pertenecientes a la empresa, resulta complejo que ésta pueda dictaminar acciones hacia trabajadores que pertenecen a otra firma. Esta situación nos indica que al implementarse una política pública debe considerarse un principio de realidad, ya que en este caso, la empresa mandante carece de facultades para imponer disposiciones a las empresas contratistas sobre las condiciones laborales de sus trabajadores. ${ }^{9}$

En relación a los trabajadores y trabajadoras, éstos se muestran satisfechos con las políticas de la compañía para conciliar la vida familiar y laboral. Como es una empresa internacional, tiene altos estándares en materia de equidad de género, superiores a las firmas chilenas. En las declaraciones de los beneficiarios/as directos/as se aprecia una gran conformidad con la empresa en las materias estudiadas, pero esta apreciación positiva no es efecto de la implementación del programa, sino de las propias políticas sensibles al género que la compañía transnacional exhibe. Como la empresa en general presenta altos estándares, puede afirmarse que ésta cumple en materia de conciliación familia-trabajo con una equidad de género. Sin embargo tal equidad de género no es resultado de la implementación del programa IGUALA.

En resumen, la implementación de las medidas para conciliar la vida familiar y laboral por la compañía minera se limitó a informar a sus trabajadores sobre esta materia. Hubo una serie de obstáculos que tornaron impracticable la aplicación de las acciones concretas, resaltando en particular que la empresa no estaba autorizada para realizar cambios en sus políticas internas. Ante estas dificultades, queda en evidencia que las acciones concretas tendientes a conciliar la vida familiar y laboral, consignadas en el programa IGUALA, no pudieron ser implementadas por la compañía minera en estudio, y que la satisfacción que trabajadoras y trabajadores presentan no es un efecto real del programa, sino de las políticas internas de la compañía. 


\section{Notas}

${ }^{1}$ El presente artículo es producto de una investigación más amplia titulada "Conciliación entre vida familiar y laboral en una empresa minera en la Región de Tarapacá”, proyecto DI 0008-09 financiado por la Universidad Arturo Prat. Una versión preliminar fue presentada en el VII Congreso de la Asociación Latinoamericana de Sociología del Trabajo, realizado en Sao Paulo del 2 al 5 de Julio de 2013.

${ }^{2}$ Ansoleaga y Godoy (2013) realizan un interesante estudio sobre los discursos actuales de la maternidad y el trabajo en Chile.

${ }^{3}$ La muestra de los beneficiarios no pretende ser representativa. Se accedió a las personas entrevistadas siguiendo el método de bola de nieve y se entrevistó a quienes mostraron disponibilidad para participar en el estudio.

${ }^{4}$ Téngase presenta la crítica realizada por académicas feministas en Europa Occidental a Esping-Andersen, quien en sus primeros análisis de los Estados de Bienestar no consideraba la contribución de las mujeres a la economía (Lewis, 1992; Ostner, 1994).

${ }^{5}$ Este programa sentó el precedente para la NCh 3262 Sistemas de Gestión igualdad de género y conciliación de la vida laboral, familiar y personal. Sello IGUALA Conciliación, que se aprobó el año 2012 y que recién ahora algunas empresas están comenzando a incorporar.

${ }^{6}$ En la región de Tarapacá el programa IGUALA se implementó el año 2009 en forma piloto en tres empresas, dos del sector minero y una del sector servicios de salud. Posteriormente se unió una cuarta empresa del sector comercial.

${ }^{7}$ El nombre de la compañía minera, así como los nombres de los entrevistados, ha sido sustituido por uno ficticio para resguardar el anonimato.

${ }^{8}$ En la minería el número de trabajadores de las empresas mandantes suele ser menos de la mitad que la cantidad de trabajadores subcontratados. Según el Reporte Anual del Consejo Minero 2013 (Consejo Minero, 2014), la compañía minera en estudio tenía aproximadamente un $40 \%$ de trabajadores propios.

${ }^{9}$ Salvo las condiciones previstas en la ley de subcontratación, que otorga a las empresas mandantes la potestad para supervigilar el pago de imposiciones de las empresas contratistas a los trabajadores que se desempeñan en la obra de la mandante. 


\section{Bibliografía}

Abarca, Nureya y Errázuriz, Margarita María (2007), "Propuestas para la Conciliación Trabajo y Familia”, en Gobierno de Chile / Pontifica Universidad Católica: Camino al Bicentenario. Propuestas para Chile, PUC, Santiago de Chile.

Aguirre, Rosario (2007), "Los cuidados familiares como problema público y objeto de políticas”, en Arriagada, Irma (coord.), Libros de la CEPAL núm. 96 Familias y políticas públicas en América Latina: una historia de desencuentros, CEPAL / UNFPA, Santiago de Chile.

Ansoleaga, Elisa y Godoy, Lorena (2013), “La maternidad y el trabajo en Chile: Discursos actuales de actores sociales”, en Polis $N^{\circ} 35$, Polis Revista Latinoamericana, Santiago.

Arriagada, Irma (2007), “Diez propuestas para mejorar la institucionalidad pública y las políticas hacia las familias en América Latina”, en Arriagada, Irma (editora) Serie Seminarios y conferencias Núm. 49, Gestión y financiamiento de las políticas que afectan a las familias, División de Desarrollo Social, CEPAL, Santiago de Chile.

Ídem (2007), “Transformaciones familiares y políticas de bienestar en América Latina”, en Arriagada, Irma (compiladora) Libros de la CEPAL núm. 96 Familias y políticas públicas en América Latina: una historia de desencuentros, CEPAL / UNFPA, Santiago de Chile.

Astelarra, Judith (2011), “Cohesión Social y familia”, en María Nieves Rico y Carlos Maldonado, Serie Seminarios y conferencias núm. 61, Las familias latinoamericanas interrogadas. Hacia la articulación del diagnóstico, la legislación y las políticas, División de Desarrollo Social, CEPAL / UNFPA.

Batthyany, Karina (2009), “Autonomía de las mujeres y resistencias a la división sexual del trabajo al interior de las familias”. En Seminario Regional Las Familias Latinoamericanas interrogadas. Hacia la articulación del diagnóstico, la legislación y las políticas. CEPAL, 29 y 30 de Octubre 2009, Santiago de Chile.

Calderón, Coral (coord.) (2013), “Redistribuir el cuidado”, en Calderón, Coral (coord.) Cuadernos de la CEPAL, núm. 101 El desafío de las políticas, CEPAL, Santiago de Chile.

Callaway, Rhonda y Harrelson-Stephens, Julie (2007), Exploring International Human Rights: Essential Readings, Lynne Rienner Publishers, Boulder.

CEPAL (2012), Panorama Social de América Latina, CEPAL, Santiago de Chile. 
Consejo Minero (2014), Reporte Anual del Consejo Minero 2013, Consejo Minero, Santiago de Chile.

Duncan, Simon y Pfau-Effinger, Birgit (2000), Gender, Economy and Culture in the European Union, Routledge, Londres.

Esping-Andersen, Gosta (1990), The Three Worlds of Welfare Capitalism, Polity Press, Cambridge.

Ídem (1999), Social Foundations of Postindustrial Economies, Oxford University Press, Oxford.

Ídem (2002), “A New Gender Contract”, en Esping-Andersen et al. (eds.), Why We Need a New Welfare State, Oxford University Press, Oxford.

Lamaute-Brisson, Nathalie (2013), “Redistribuir el cuidado: para un nexo de políticas públicas”, en Calderón, Coral (coord.) Cuadernos de la CEPAL, núm. 101 Redistribuir el cuidado. El desafío de las políticas, CEPAL, Santiago de Chile.

Lamus, Doris (2008), “La Agenda Global de las Naciones Unidas para "la Mujer” ”, en Polis $N^{\circ} 20$, Polis Revista Latinoamericana, Santiago.

Leiva, Sandra (2009), Políticas públicas y genderfare: conciliando la vida familiar y laboral, Ponencia presentada al $21^{\circ}$ Congreso Mundial de Ciencia Política realizado por la Asociación Internacional de Ciencia Política, Santiago de Chile, 12 al 16 de julio de 2009.

Ídem (2012), “Trabajadores subcontratados en la minería del cobre en Chile”, en Revista Trabajo $N^{\circ}$ 9, Universidad Autónoma Metropolitana y Organización Internacional del Trabajo, México D.F.

Leiva, Sandra y Campos, Alí (2013), “Movimiento social de trabajadores subcontratados en la minería privada del cobre en Chile”, en Revista Psicoperspectivas, Vol. 12, $N^{\circ}$ 2, pp. 51-61, Valparaíso.

Lewis, Jane (1992), “Gender and the Development of Welfare Regimes”, Journal of European Social Policy, 2, 3, pp. 159-173.

Martínez, Juliana y Camacho, Rosalía (2007), “Equilibristas o malabaristas..., pero ¿con red? La actual infraestructura de cuidados en América Latina” en Carbonero, María Antonia y Levín, Silvia Entre Familia y Trabajo. Relaciones, conflictos y políticas de género en Europa y América Latina, Homo Sapiens Ediciones, Rosario.

Montaño, Sonia (2010), “El cuidado en acción”, en Montaño, Sonia y Coral Calderón (coordinadoras) Cuadernos de la CEPAL núm. 94 El cuidado en acción. Entre el derecho y el trabajo, CEPAL / Agencia Española de Cooperación Internacional para el Desarrollo (AECID) / Fondo de Desarrollo de 
las Naciones Unidas para la Mujer (UNIFEM), Santiago de Chile.

Orellana, Víctor (2010), Evaluación y Sistematización Programa de Buenas Prácticas Laborales con Equidad de Género. Síntesis Ejecutiva. Documento Servicio Nacional de la Mujer, Santiago de Chile.

Organización Internacional del Trabajo (2009), Trabajo y Familia: Hacia nuevas formas de conciliación con corresponsabilidad social, OIT / PNUD, Santiago de Chile.

Ostner, Ilona (1994), “The Women and Welfare Debate”, en L. Hantrais y S. Morgan (eds.), Cross-National Research Papers, Third Series, 3 Family Policy and the Welfare of Women, European Research Centre, Universidad de Loughborough.

Pautassi, Laura (2010), “Cuidado y derechos: la nueva cuestión social”, en Montaño, S. y Calderón, C. (coords.) El cuidado en acción: entre el derecho y el trabajo, CEPAL, Santiago de Chile.

Pautassi, Laura y Rico, María Nieves (2011), “Licencias para el cuidado infantil. Derecho de hijos, padres y madres”, en Desafíos Número 12, pp. 4-9, CEPAL / UNICEF, Santiago de Chile.

Rico, María Nieves (2011), “Crisis del cuidado y políticas públicas: el momento es ahora”, en Rico, María Nieves y Maldonado, Carlos, Serie Seminarios y conferencias núm. 61, División de Desarrollo Social, Las familias latinoamericanas interrogadas. Hacia la articulación del diagnóstico, la legislación y las políticas, CEPAL / UNFPA.

SERNAM (2003), Análisis de los costos y beneficios de implementar medidas de conciliación vida laboral y familiar en la empresa, Documento de Trabajo N 84, SERNAM, Santiago de Chile.

Ídem (2008), Código de Buenas Prácticas Laborales con Equidad de Género, SERNAM, Santiago de Chile. 